\title{
Simulation Based Approach for the Industrialization of a Cabinet Manufacturing Facility
}

\author{
Ryan Brown ${ }^{1 *}$, Chelsea Ritter ${ }^{2}$, and Mohamed Al-Hussein ${ }^{3}$ \\ ${ }^{1}$ M.Sc. Student, Department of Civil and Environmental Engineering, University of Alberta \\ ${ }^{2}$ PhD Student, Department of Civil and Environmental Engineering, University of Alberta \\ ${ }^{3}$ Professor, Department of Civil and Environmental Engineering, University of Alberta \\ *Corresponding author's e-mail: rabrown1@ualberta.ca
}

\begin{abstract}
High-end cabinet making is traditionally an artisan process that utilizes few manufacturing principles. Manufacturing lead time, labor hours required, and productivity can be improved by industrializing the process. This paper focuses on a case study of a high-end cabinet manufacturer in Edmonton AB, Canada and the proposed process and facility improvements. First, computer simulation using Simphony.NET and movement analysis of people/materials of the cabinet manufacturer's current state of operations is conducted to establish a baseline. Next, suggested process and facility layout improvements and their anticipated results are quantified through future state simulation in order to aid management in making decisions for plant changes and to prove their effectiveness. These improvements include: application of lean principles, modification of their current production methods to reduce bottlenecks, and future state facility layout based on an optimized flow of people and materials.
\end{abstract}

\section{KEYWORDS}

Cabinet Manufacturing; Discrete Event Simulation; Process Improvements; Lean Principles, Facility Layout

\section{INTRODUCTION}

Industrialization of cabinet production by shifting operations from traditional methods into a manufacturing format can achieve productivity and lead time benefits while reducing labor hour requirements. A major tool used to industrialize a process is the use of lean methodologies. Lean production is an approach used to improve manufacturing efficiency by continuously eliminating wastes in its many forms. This production philosophy improves competitiveness by identifying and eliminating waste (non-value adding) activities (Koskela, 1992). In this case study, waiting, transportation, and motion wastes are focused on since they are the most prominent in the facility. Lean emphasizes the consideration of flow and value generation in designing construction processes in order to achieve a lean process (Abbasian-Hosseini et al. 2014). By identifying which processes add value to the customer, lean seeks to optimize, continuously improve, and eliminate waste in this value stream. The successful implementation of lean principles increases efficiency, productivity, and quality in the workplace (Kobayashi and Fisher, 2008). Typical benefits of converting from traditional production to fully lean manufacturing can be dramatic and have been documented in a variety of industries (Manufactured Housing Research Alliance, 2007). Examples 
include the lean transformation of a modular building company that resulted in reduced labor costs and improved labor efficiency (Yu et al., 2013). Another is the improvement of cycle time, productivity, and process efficiency by applying the same principles to a bricklaying process (Abbasian-Hosseini et al. 2014). The implementation of lean principles gives rise to a facility layout problem (FLP). FLP is defined as the placement of workstations within the facility, with the aim of determining the most effective arrangement in accordance with some criteria or objectives under certain constraints (Hosseini-Nasab et al., 2017). In order to eliminate transportation and motion wastes, the workstations must be setup logically to provide the best flow from station to station. To eliminate waiting, each station must be standardized to be task specific to prevent a backlog of different tasks needing to be performed at the same workstation. To eliminate these wastes a process-orientated future state facility layout with standardized workstations is required to solve the FLP and achieve lean production. While lean principles are used to identify potential process improvements within the manufacturing facility, these improvements should be tested and validated in an environment before being implemented on the manufacturing floor. Simphony.NET is an integrated environment for simulating construction activities that was developed by AbouRizk and Mohamed (2000). Simulation models are used to replicate complex operations and to test potential production line improvements. Discrete event simulation (DES) is a cost-effective method to test these changes before they impact the factory floor and is an effective decision support tool that allows people to precisely examine different approaches in order to complete a project in the most efficient manner (Altaf et al. 2015). By simulating proposed process improvements, management can easily judge which changes to implement and the effect that these proposed process improvements will have on reaching company goals.

\section{Motivation}

The case study company is a high-end cabinet manufacturer located in Edmonton, Alberta, Canada. The manufacturer has grown significantly over the last few years and has recently moved into their current facility, with another planned move in approximately four years. To manage the increased production volume, the company seeks to adapt its manufacturing process in order to maintain their strong focus on delivering high quality cabinets. The manufacturer aims to accomplish three specific goals: (1) to decrease their manufacturing lead time by $30 \%$; (2) to decrease the number of labor-hours required for production by $15 \%$; and (3) to increase their productivity by $24 \%$. This paper outlines the process and facility layout improvements proposed to transform current production operations into a more efficient overall manufacturing process that meets the three specified goals. These goals are necessary for the case study company to continue growing into their current facility and to provide a road map of how their future facility should be set up. By achieving these goals, the cabinet manufacturer will be able to maintain their focus on delivering high quality cabinets and continue to prosper.

\section{METHODS}

\section{Current State Manufacturing Process}

The first step in meeting the manufacturing lead time, labor-hour, and productivity goals is to quantify their current operations and establish a baseline. This is done by studying current operations to identify the flow of material and people throughout the plant. Next, a time study is completed, and a simulation model is built and validated. 
Current State Production. The current production process is shown in Figure 1. This flow chart illustrates the main stations used to produce a melamine cabinet box with either wood or polylaminate (P-Lam) faces. The production process for melamine cabinet boxes is as follows: (1) a CNC machine cuts the sheets to the desired size; (2) an edgebanding machine is used to put edges on to cut pieces; (3) the pieces are assembled to form the cabinet box. The process for drawer boxes is the same, except for drawer hardware installation between steps (2) and (3). For polylaminate cabinet faces the operations are as follows: (1) polylaminate sheets are glued onto both sides of particle board to create the sheet material; (2) a CNC machine cuts the sheets to the desired size; (3) an edgebanding machine is used to put edges on to cut pieces; (4) door hardware is installed; (5) the faces are hung onto the cabinet box. For wood faces, the process is as follows: (1) a beam saw cuts sheets to the desired size; (2) unfinished faces are hung on cabinet boxes to check size and alignment; (3) sanding is done three times-before the 1st coat of paint, and after the 1st and 2nd coat; (4) painting is done after each sanding; (5) door hardware is installed; (6) the faces are hung onto the cabinet box.

Current State Simulation Model. This production flow and data from the time study is used to build the current state simulation model (Figure 2). The simulation model was developed through discrete event simulation in Simphony.NET, a program developed by AbouRizk and Mohamed (2000). The current state simulation model was constructed by representing each workstation as an entity. These entities are further broken down into sub-entities to model the individual tasks being performed at each workstation. For each of the tasks, a distribution or average timing was used since the timings varied for each project and person. If enough observations were recorded (approximately $\geq 10$ ), Simphony.NET was used to find the best fitting distribution, an average time was used for the tasks without enough observations to fit a distribution to. Constant values were used for the glue up, wrapping, and project quality control task timings only, since they were assumed to be relatively fixed. It is important to note that the simulation model focuses on task times involved with productive activities only and not the time taken in between stations.
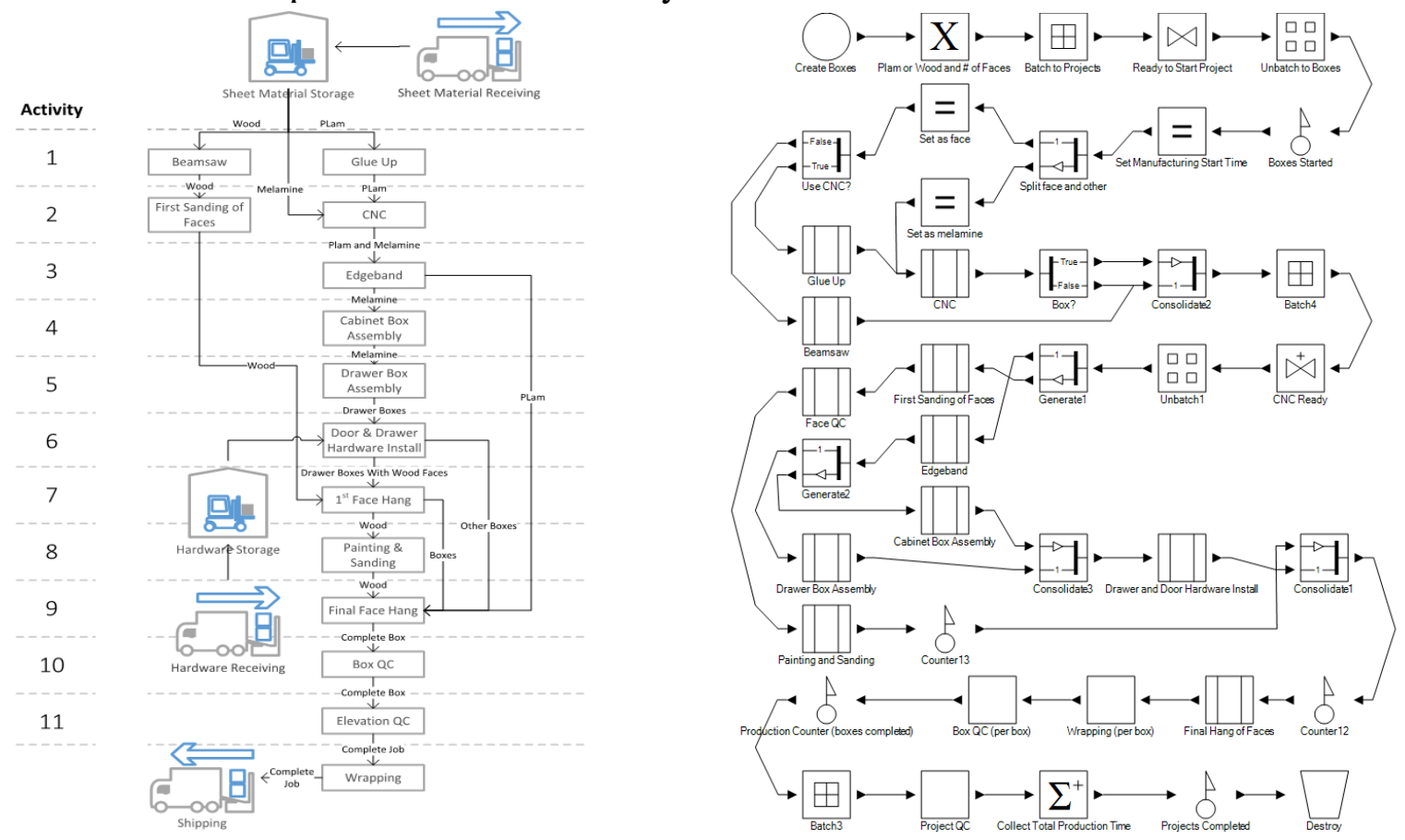
Figure 1. Current State Production Process $\quad$ Figure 2. Current State Simulation Model Validation and Verification of the Current State Simulation Model. The simulation model was validated based on time estimates, project totals, and material usage estimates provided by the case study company from previous studies of their own. The validation of the sheet material usage can be seen in Table 1. A 5\% error on the number of boxes simulated in the test run year (608 boxes were created in the simulated year and 640 in the sample year provided by the company) was also calculated but is considered acceptable as only one year is being used and is available as a reference. Five workers were used in the simulation, each with 5.5 hours of time available each day (due to coffee breaks, meetings, cleaning, and other tasks), and 227 working days per year. A new project was made available to the plant floor every 20 to 25 days, approximately.

Table 1. Current State Simulation Model Material Usage Validation

\begin{tabular}{|c|c|c|c|}
\hline & Melamine $\left(\mathbf{f t}^{\mathbf{2}}\right)$ & Wood $\left(\mathbf{f t}^{\mathbf{2}}\right)$ & P-Lam $\left(\mathbf{f t}^{\mathbf{2}}\right)$ \\
\hline $\begin{array}{c}\text { Estimate based on data from the case } \\
\text { study company }\end{array}$ & 34,857 & 2,646 & 2,484 \\
\hline Simulation Result & 32,589 & 2,710 & 2,506 \\
\hline Percent Error & $7 \%$ & $2 \%$ & $1 \%$ \\
\hline
\end{tabular}

\section{Process Improvements}

Numerous process improvements have been proposed to meet the three goals by targeting areas of inefficiency and bottlenecks. The methods used alter current processes to reduce task durations based on lean principles and change the sequence of production to allow for better flow during manufacturing. The corresponding estimated cost level of implementation is listed in Table 2 and the changes are described in detail below.

Table 2. Estimated Cost of Implementation

\begin{tabular}{|l|l|}
\hline Process Improvement & Cost \\
\hline Kits Made for Cabinet and Drawer Boxes & Low \\
\hline Reduce Box Quality Control Time & Low \\
\hline Reduce Edgeband Setup Time & Low \\
\hline Reduce Sanding Preparation Time & Low \\
\hline Quality Control Step to Replace First Face Hang & Low \\
\hline Cabinet and Drawer Boxes Assemblies in Parallel & Low \\
\hline Hardware Ready & Low \\
\hline Improve Wrapping & Low - Medium \\
\hline Reduce Painting Setup Time & Low - Medium \\
\hline Reduce Walking Time & Low - Medium \\
\hline Premade Cut Plans & Medium \\
\hline Reduce Drying Time & High \\
\hline
\end{tabular}

Reduction of Task Durations. Multiple opportunities for improving task times were found throughout the production process; these changes range from minor organizational fixes to major additions to the plant. First, the edgeband setup time can be reduced through better organization of the pieces coming from the cutting area. This will allow more pieces to be completed without 
adjustment of the machine. The setup time will reduce from an average of 2 minutes per box to an average of 30 seconds per box. A reduction of the time required for sanding preparation can be accomplished by the addition of a dedicated sanding table and drying area. A dedicated drying area will allow for better flow from the paint booth into the drying area and then to the sanding table. A sanding table will also make the required tools readily available for the dried pieces. This should cut the sanding preparation time in half to be 1 min per box per coat. By improving the organization of the paint booth and reducing the batch size of pieces into the paint booth, the painting setup time can be reduced. Having carts that are less full and having more space to move pieces along, along with having the drying area organized, will make it much easier to get set up for painting. It is estimated that the painting setup time can be reduced by about 45 seconds per box. The quality control time is reduced by labelling the boxes and placing them in a designated staging area. It is likely that this improvement will cut time from 5 to 2.5 minutes per box since all components to be inspected will be easily locatable and identifiable. Reduction of the drying time is achieved through the addition of a full drying chamber. This change would have a major effect on production since drying time would be reduced to 0.5 hours from 2 to 3 hours per coat. However, this is a high cost item and a large change to the plant. By producing "kits" for cabinet and drawer boxes, the pieces need for cabinet and drawer box assemblies can be better organized. Instead of pieces being places on a cart after the CNC based on their size, they will be placed according to which cabinet or drawer box they belong to. These kits will be coordinated with hardware packages before being delivered to the cabinet and drawer box assembly lines. Having hardware ready for door and drawer assembly will improve assembly time since all components will be delivered to the correct workstations, rather the person constructing the box needing to go to collect it. Premade cut plans for the $\mathrm{CNC}$ and beamsaw will save time by freeing the operator from manually programming the saws. This change reduced the time from 4 minutes per sheet to design the cuts for the $\mathrm{CNC}$ and 3 minutes per sheet to enter the cut info on the beamsaw to 1 minute per sheet for each task. Finally, a lazy-susan style wrapping turn table or an automated turn table with a height-adjustable stretch wrap holder should be purchased to improve the wrapping of finished cabinets. This will reduce the hand wrapping time of 10 minutes per box to 1 minute per box.

Changing Production Sequence. An analysis of the current state process detailed in the process flow diagrams in Figure 1 shows that the critical path for an average cabinet box to be completed would be about 606.5 minutes. Most of the precedence relationships cannot be changed, but one that can, is the quality control (QC) for the wood cabinet faces. The introduction of a 10-minute QC measure instead of the first face hang would allow a change of the precedence relationships that would make the critical path for the average cabinet 552.7 minutes. The manufacturing lead time can be further shortened by using the parallel production lines to assemble the cabinet and drawer boxes at the same time as opposed to one after another. No task times are changed in this estimate, but flow through these stations will be improved.

\section{Facility Layout}

The current facility layout has a general flow of materials based on the location of the larger machines and the shipping and receiving doors. In this current layout, workstations are based on the individual working there rather than standardizing for the task being performed. This personspecific workstation approach creates an unpredictable flow, increases wasted transportation, and is a root cause of disorganization in the plant. These three problems can be solved by designing a 
future state facility layout that focuses on plant flow, minimum walking distances, and the use of standardized workstations.

Reduce Walking Time. A movement analysis was done through "spaghetti diagrams" to determine the percent time spent walking during a specific task. Workers were observed and timed as they performed tasks and their walking paths were traced. Using an average walking speed, total time, and the total distance traveled, an average of $10 \%$ of wasted movement during tasks was calculated. Under the revised plant layout, this time wasted walking can be instead shifted to productive time to determine the possible productivity gain from reducing wasted movement.

Task Centered Workstations. The current cabinet production process is centered around individual work stations, where each person performs their duties. This type of production creates an uneven use of space and an uncertain flow of materials and people through the plant. This setup also increases the number of tools required at each station, as there is a larger variety of possible jobs that could be done at each workstation. Efficiency can be gained by having set workstations based on the activity being done. These activities include drawer assembly, cabinet assembly, final assembly, sanding, drying, staging, and special projects.

Future State Facility Layout. The future state facility layout is based on the current state production flow but incorporates walkways, staging and drying areas, improved material transport, rearranging of stations, and specialized workstations. The addition of a painted walkway will ensure indicated areas are kept clear of material to allow for people and products to flow unobstructed through the plant and from station to station. Moving of the special projects' benches and glue up stations will place these stations in a more logical position to increase plant flow and reduce wasted movement. A designated drying and sanding area allows for a cyclical flow of carts from the paint booth to the sanding table, then back to the paint booth if additional coats are needed, thus increasing plant flow and keeping the plant floor unobstructed. A designated staging area will provide an area for all projects to be set up for QC. This will help to keep the pathways clear, as currently staging of projects often blocks passages in the current plant setup. The most major change is the task centered workstations: this change is vital to the success of the future state layout. These numerous changes help to create a natural and predictable flow of people and materials through the plant. Improved organization, a reduction of wasted movement and transportation, and increased standardization and safety are also gained.

\section{RESULTS AND DISCUSSION}

\section{Future State Simulation}

Several process improvements and an improved facility layout have been proposed in order to improve the manufacturing lead time, labor hours required, and productivity. These improvements and their corresponding effect on reaching the three goals are specified in Table 3. These results were found by applying each process improvement individually to the base case. The total effect of all improvements is then modelled to find the cumulative effect of these changes. It is important to note that the sum of the individual changes is not the same result as applying all the changes at once. This is due to resource constraints in the simulation model for the number of workers, machines, and workstations. 
Many of the process improvements are low cost and represent slight changes to production. These should be implemented first because they can be done with little disruption while still having a significant impact on lead time, productivity, and labor hours required. The higher cost and more major plant changes, such as purchasing of equipment or significantly altering the production steps, can be implemented once resources are available and management and employees have discussed when and how they will apply these changes. However, all the process improvements discussed are necessary to achieve the desired three goals. All the suggested process improvements were presented to the cabinet manufacturers management team to ensure that the changes are realistic and feasible for their plant. The feedback received was positive and the facility aims to implement these changes over time as people and resources become available. The priority of the changes will be based on lowest cost and least disruption to the plants operation. Most of suggested changes will be implemented in their current plant, while the purchasing of a full drying chamber is likely to be done once they move into their new facility.

Table 3. Future State Simulation Results

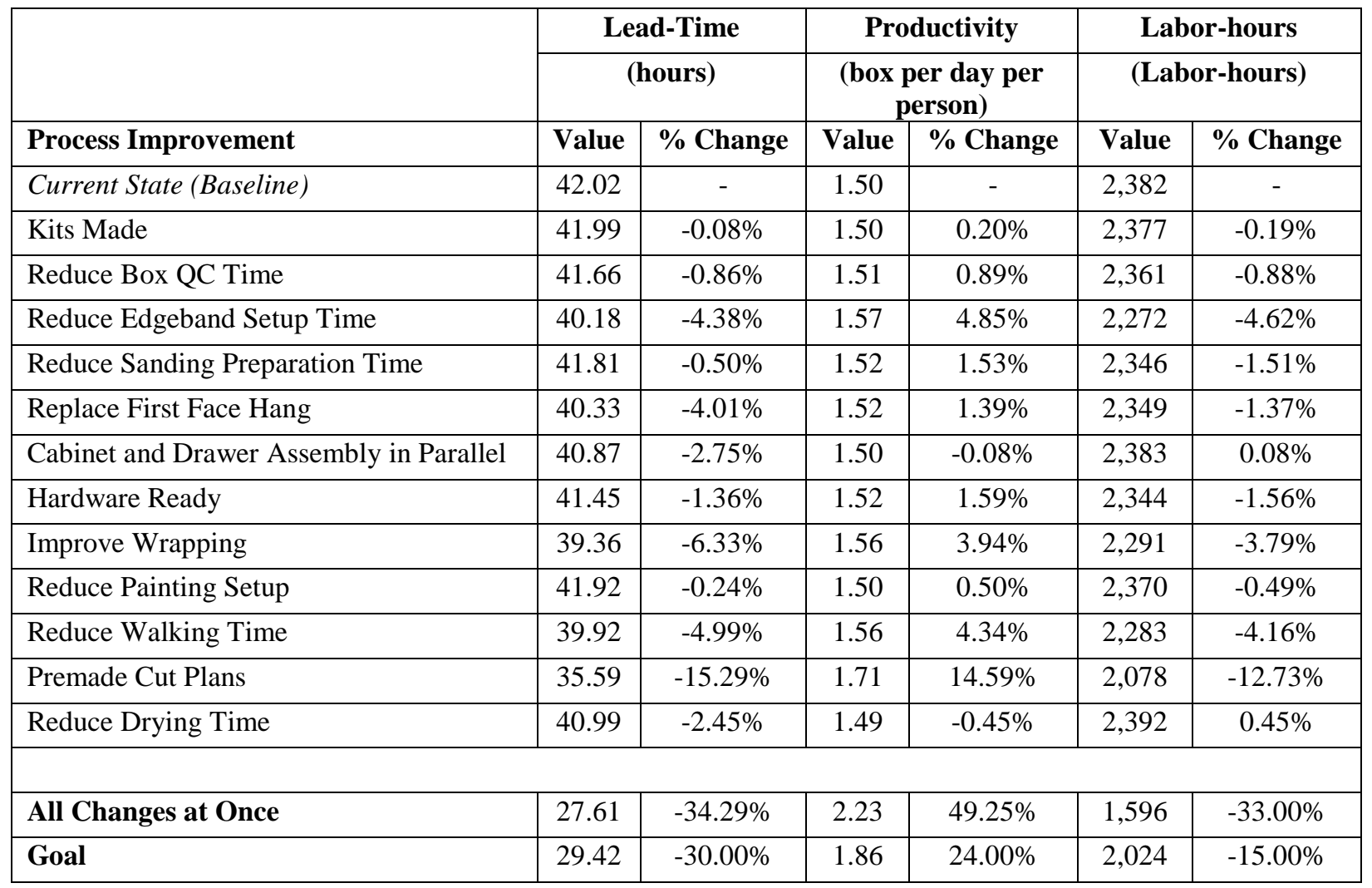

\section{CONCLUSION}

By implementing the numerous changes outlined in this report, it is possible for the cabinet manufacturer to reduce lead time and labor hours required by $34 \%$ and $33 \%$, respectively, and to increase productivity by $49 \%$. Many changes are found to be low cost and require only a slight change to production, while others are costlier and more disruptive to operations. The implementation of all these changes was found to produce significant benefits for the plant. Once most changes have been completed, production should be further evaluated through time studies 
to confirm assumptions and time saving estimates. Using the time data, bottlenecks and areas of inefficiency can be identified for future improvements. This research only included timings for productive tasks, while a large part of the time spent was on non-productive tasks. A future study of non-productive time may be beneficial to identify causes and help shift this into productive time. Limited observations were a factor during this research; therefore, additional task timings would be valuable to fit all task durations to a distribution rather than using an average or constant value for the tasks with limited observations.

\section{ACKNOWLEDGEMENTS}

The authors would like to thank everyone at the case study company in Edmonton, AB, Canada, for allowing us to observe their actions at the facility in order to collect the data needed for this study. We would like to specifically thank the owner and manager of the case study company for organizing visits and providing information. Additional thanks to Riphay Al-Hussein and Scott Penner for organizing this project. The research is funded by Natural Sciences and Engineering Research Council of Canada Engage Grant (EGP 532044-18).

\section{REFERENCES}

Abbasian-Hosseini, S., Nikakhtar, A. and Ghoddousi, P. (2014). "Verification of lean construction benefits through simulation modeling: A case study of bricklaying process." KSCE Journal of Civil Engineering, 18(5), 1248-1260.

AbouRizk, S., and Mohamed, Y. (2000). "Simphony-an integrated environment for construction simulation." 2000 Winter Simulation Conference Proceedings (Cat. No.00CH37165), 2, 1907-1914.

Altaf, M.S., Liu, H., Zhang, Y., Al-Hussein, M. and Bouferguene, A. (2015) "Discrete-Event Simulation Modelling of Prefabricated Wall Production Line.” Proceedings, European Modeling and Simulation Symposium, Bergeggi, Italy, 234-239.

Hosseini-Nasab, H., Fereidouni, S., Fatemi Ghomi, S. and Fakhrzad, M. (2017). "Classification of facility layout problems: a review study." The Int. Journal of Adv. Manufacturing Technology, 94(1-4), 957-977.

Kobayashi, K., Fisher, R. (2008). "Business improvement strategy or useful tool? Analysis of the application of the $5 \mathrm{~S}$ concept in Japan, the UK and the US." <https://doiorg.login.ezproxy.library.ualberta.ca/10.1080/14783360701600704> (Nov. 25, 2018).

Koskela, L. (1992). Application of the new production philosophy to construction (Vol. 72). Stanford, CA, USA.

Manufactured Housing Research Alliance (2007). "Pilot Study: Applying Lean principles to Factory Home Building." <https://www.huduser.gov/portal/Publications/pdf/pilotstudy.pdf> (Nov. 20, 2018).

Yu, H., Al-hussein, M., Asce, M., Al-jibouri, S., and Telyas, A. (2013). "Lean Transformation in a Modular Building Company: A Case for Implementation." Journal of Management in Engineering, 29(January), 103-111. 